\title{
UNIQUENESS CRITERIA FOR SOLUTIONS OF SINGULAR BOUNDARY VALUE PROBLEMS
}

\author{
BY \\ D. R. DUNNINGER AND HOWARD A. LEVINE( ${ }^{\mathbf{1}}$ )
}

ABSTRACT. In this paper we consider the equation

$$
u^{\prime \prime}(t)+(k / t) u^{\prime}(t)+A u(t)=0, \quad 0<t<T, \quad u(T)=0,
$$

where $u:(0, T) \rightarrow D(A) \subset B$ is a Banach space valued function taking values in a dense subdomain $D(A)$ of the Banach space $B$. Here $A$ is a closed (possibly unbounded) linear operator on $D(A)$ while $k$ is a real constant. The differential equation is an abstract Euler-Poisson-Darboux equation. We give necessary and sufficient conditions on the point spectrum of $A$ to insure uniqueness of the strong solution $u \equiv 0$ as well as sufficient conditions on the point spectrum to insure uniqueness of weak solutions. $u$ is only required to satisfy

(a) $t^{k}\left\|u^{\prime}(t)\right\| \rightarrow 0^{+}$as $t \rightarrow 0^{+}$if $k>1$,

(b) $t^{k}\left\|u^{\prime}(t)\right\|+t^{k+1}\|u(t)\| \rightarrow 0$ as $t \rightarrow 0^{+}, 0<k<1$,

(c) $t\left\|u^{\prime}(t)\right\|+\|u(t)\| \rightarrow 0$ as $t \rightarrow 0^{+}, k<0$.

The operator $A$ need not possess a complete set of eigenvectors nor need one have a backward uniqueness theorem available for (1) for the Cauchy final value problem.

Our techniques extend to the $\boldsymbol{n}$-axially symmetric abstract equation

$$
\sum_{i=1}^{n}\left[\partial^{2} u / \partial t_{i}^{2}+\left(k_{i} / t_{i}\right) \partial u / \partial t_{i}\right]+A u=0 .
$$

The proofs rest upon an application of the Hahn-Banach Theorem and the consequent separation properties of $B^{*}$, the dual of $B$, as well as the completeness properties of the eigenfunctions of certain Bessel equations associated with (1).

I. Introduction. In an earlier paper [7], the authors gave necessary and sufficient conditions for the boundary value problem

$$
\begin{gathered}
d^{2} u / d t^{2}+A u(t)=0, \quad 0<t<T<\infty, \\
u(0)=u(T)=0,
\end{gathered}
$$

to have a unique solution. Here $u:[0, T] \rightarrow D(A)$ is a twice strongly continuously differentiable function (in the norm of a Banach space $B$ ) and $D(A) \subset B$ is the domain of the given closed operator $A$. The results were extended in several directions there to include operator equations of the form

Received by the editors March 13, 1975.

AMS (MOS) subject classifications (1970). Primary 35Q05, 35L20, 35R25; Secondary 35M05, 34B99.

$\left({ }^{1}\right)$ Supported in part by NSF Grant no. GP42677. 


$$
P(t) u(t)+A u(t)=0,
$$

where $P(t)$ was an even order ordinary differential operator with (possibly unbounded but time independent) operators as coefficients. Several applications to boundary value problems for partial differential equations were given. We refer the reader to [7] for details and a more comprehensive bibliography.

The purpose of this paper is twofold. First,we examine boundary value problems for an abstract Euler-Poisson-Darboux (EPD) equation of the form

$$
d^{2} u / d t^{2}+(k / t) d u / d t+A \mu=0, \quad 0<t<T<\infty,
$$

where $k$ is a given real constant, $k \neq 0$.

Equation (1.3) includes, as special cases, both the classical EPD equation as well as the generalized axially symmetric potential theory equation (GASPT). More generally, it includes equations of the form

$$
\frac{\partial^{2} u}{\partial t^{2}}+\frac{k}{t} \frac{\partial u}{\partial t}-\sum \frac{\partial}{\partial x_{i}}\left(a_{i j}(x) \frac{\partial u}{\partial x_{j}}\right)=0, \quad(x, t) \in \Omega \times(0, T),
$$

where $\Omega$ is a bounded domain in $R^{n}$. The symmetric matrix $\left(a_{i j}(x)\right)$ may have both positive and negative eigenvalues.

Because the appropriate boundary conditions for (1.3) to be prescribed at $t=0$ will depend upon the parameter $k$, we shall not specify them here but include them in our discussions later.

Secondly, we shall indicate how our earlier results [7] may be extended to weak solutions of (1.1) and (1.3).

Our results extend those of Young [11], [12], Dunninger and Weinacht [8] to the case $k<0$. Since they are of an abstract nature they apply to a much wider class of problems than heretofore considered. Moreover, our techniques permit us to dispense with several conditions on the operator $A$ needed in earlier results. Among these were the following:

(a) $A$ is formally selfadjoint and elliptic.

(b) $A$ possesses a complete set of eigenfunctions.

(c) The undifferentiated term (if any) in $A$ must be nonnegative.

(d) A backward (in time) uniqueness theorem must be available for (1.4).

The plan of the paper is as follows. In the next section we formally introduce the definitions and notation to be used. In §III we examine classical (strong) solutions to (1.3) with the condition $u(T)=0$. In the next section we consider the boundary condition $\dot{u}(T)+\alpha u(T)=0, \alpha$ real. Then, in $\S \mathrm{V}$ we consider weak solutions to (1.1) and (1.3). Finally, we mention, in §VI, the abstract biaxially symmetric equation. 
II. Definitions and notation. Throughout the paper we let $B$ be a real Banach space and $D(A) \subset B$ be a dense linear subspace. $A: D(A) \rightarrow B$ will be a given closed linear operator, which need not be bounded in general. The restriction that $B$ be real is made only for convenience of notation as can easily be seen from the results and their proofs. Let $B^{*}$ denote the dual of $B$ and $\langle f, x\rangle$ denote the pairing of $f \in B^{*}$ with $x \in B$. In $\S \mathrm{V}$ we will have need of the adjoint of $A, A^{*}$. Let

$D\left(A^{*}\right)=\left\{f \in B^{*} \mid\langle f, A x\rangle\right.$ is a continuous linear functional of $\left.x \in D(A)\right\}$.

$D\left(A^{*}\right)$ thus is the domain of the adjoint operator $A^{*}: D\left(A^{*}\right) \rightarrow B$. We shall assume that $D\left(A^{*}\right)$ is dense in $B^{*}$ (in the norm topology), but we will not use this fact except in $\S \mathrm{V}$.

Definition II-1. A sequence $\left\{\lambda_{n}\right\}_{n=1}^{\infty}$ is said to be admissible for uniqueness of strong solutions if $\lambda_{n} \notin \sigma_{p}(A)$ (the point spectrum of $A$ ) for $n=1,2$, $3, \ldots .$.

Throughout this paper we shall assume $k \neq 0$. The case $k=0$ is the regular case and has been treated in [7].

III. Strong solutions. We define a strong solution as follows:

Definition III-1. $u:(0, T] \rightarrow D(A)$ is a strong solution to

$$
d^{2} u / d t^{2}+(k / t) d u / d t+A u(t)=0, \quad t \in(0, T)
$$

if

(i) $u$ is strongly (norm) continuous on $(0, T]$.

(ii) $u$ has two weakly continuous weak derivatives $d u / d t, d^{2} u / d t^{2}$.

(iii) Equation (3.1) is satisfied in the following weak sense: for all $f \in B^{*}$, $t \in(0, T)$,

$$
\left\langle f, d^{2} u / d t^{2}\right\rangle+(k / t)\langle f, d u / d t\rangle+\langle f, A u(t)\rangle=0 .
$$

(iv) Let $\rho(t)=t^{k}\|A u(t)\|$ if $k>0, t\|A u(t)\|$ if $k<0$. Then $\rho(t)$ is integrable on $(0, T]$.

REMARK. In view of the definition of weak derivatives, the equation (3.2) means that for all $f \in B^{*},\langle f, u\rangle$ is twice continuously differentiable, and

$$
\frac{d^{2}}{d t^{2}}\langle f, u(t)\rangle+\frac{k}{t} \frac{d}{d t}\langle f, u(t)\rangle+\langle f, A u(t)\rangle=0 .
$$

Definition III-2. We say $u:(0, T] \rightarrow D$ is a strong solution to the first boundary value problem (FBVP) for (3.1) if

(i) $u$ is a strong solution to (3.1) in the sense of Definition III-1.

(ii) $u(T)=0$. 
(iii) (a) if $k>0, t^{k}[\|d u / d t\|+t\|u(t)\|] \rightarrow 0$ as $t \rightarrow 0^{+}$;

(b) if $k<0[t\|d u / d t\|+\|u(t)\|] \rightarrow 0$ as $t \rightarrow 0^{+}$.

REMARK. The conditions (iii)(a), (b) seem rather restrictive. In fact they are much weaker than the conditions used in [8], [11], [12] which required $u$ to be continuously differentiable on $[0, T]$. For $k>0$, condition (a) says that $\|d u / d t\|,\|u\|$ may grow to infinity like $t^{\epsilon-k}$ and $t^{\delta-k-1}$ for some $\epsilon, \delta, \epsilon<k$, $\delta<k+1$.

REMARK. For $k>1$, one can give an $\epsilon-\delta$ argument to show that if $t^{k}\|d u / d t\| \rightarrow 0$ as $t \rightarrow 0^{+}$, then $t^{k-1}\|u(t)\| \rightarrow 0$ and consequently $t^{k+1}\|u(t)\| \rightarrow 0$ as $t \rightarrow 0^{+} .\left(^{2}\right)$

To see this we observe that

$$
\begin{aligned}
\|u(t)\| & \leqslant\left\|u\left(t_{0}\right)\right\|+\int_{t}^{t_{0}}\|d u / d \eta\| d \eta \\
& \leqslant\left\|u\left(t_{0}\right)\right\|+(\epsilon /(k-1)) t^{-k+1},
\end{aligned}
$$

where $t_{0}>0$ is so small that $t^{k}\|d u / d t\|<\epsilon$ on $\left(0, t_{0}\right)$. From this we see that $t^{k-1}\|u(t)\| \rightarrow 0$ as $t \rightarrow 0^{+}$. Thus $t^{k+1}\|u(t)\| \rightarrow 0$ as $t \rightarrow 0^{+}$.

Now let $\left\{\lambda_{n}\right\}_{n=1}^{\infty}$ denote the eigenvalues for the following Sturm-Liouville problem for Bessel's equation of order $p$ where $p \equiv 1 / 2|k-1|$

$$
\begin{gathered}
\psi^{\prime \prime}(t)+t^{-1} \psi^{\prime}(t)+\left(\lambda-p^{2} t^{-2}\right) \psi(t)=0, \\
\psi(T)=0,
\end{gathered}
$$

$$
t^{1 / 2} \psi(t) \text { is bounded near } t=0 .
$$

If $k \geqslant 1$ or $k<0$, we take

$$
\psi_{n, p}(t)=J_{p}\left(\sqrt{\lambda}_{n} t\right)
$$

where the $\lambda_{n}$ are the positive roots of

$$
J_{p}\left(\sqrt{\lambda}_{n} T\right)=0 .
$$

The functions $\psi_{n, p}$ form a complete orthogonal sequence with respect to the weight function $t$. On the other hand, if $0<k<1$, or $0<p<1 / 2$, there are two sets of solutions:

$$
\psi_{n, p}(t)=J_{p}\left(\sqrt{\lambda}_{n} t\right) ; \quad J_{p}\left(\sqrt{\lambda}_{n} T\right)=0,
$$

and

$$
\psi_{n, p}(t)=J_{-p}\left(\sqrt{\lambda}_{n} t\right) ; \quad J_{-p}\left(\sqrt{\lambda}_{n} T\right)=0
$$

(2) The authors wish to thank Professor Richard Weinacht for this observation. 
Recall that, for any $r, r$ not a negative integer,

$$
J_{r}(t)=\frac{t^{r}}{2^{r} \Gamma(r+1)} \sum_{m=0}^{\infty} \frac{(-1)^{m} \Gamma(r+1) t^{2 m}}{2^{2 m} \Gamma(r+m+1) \Gamma(m+1)}
$$

From now on, we shall write $\psi_{n}$ instead of $\psi_{n, p}$ or $\psi_{n,-p}$ and mean either the sequence given by (3.5) or (3.7).

THEOREM III-1. Let $u:(0, T] \rightarrow D$ be a strong solution to the FBVP. Then $u \equiv 0$ if and only if the sequence $\left\{\lambda_{n}\right\}_{n=1}^{\infty}$ defined by (3.5.1) or (3.7) is admissible for uniqueness in the sense of Definition II-1.

Proof. Suppose for some $m, \lambda_{m}$ is an eigenvalue for $A$. Let

$$
u_{m}(t)= \begin{cases}t^{1 / 2(1-k)} J_{p}\left(\sqrt{\lambda}_{m} t\right) v_{m}, & k \geqslant 1 \text { or } k<0 \\ t^{1 / 2(1-k)} J_{-p}\left(\sqrt{\lambda}_{m} t\right) v_{m}, & 0<k<1,\end{cases}
$$

where $v_{m} \in D(A), v_{m} \neq 0$, is an eigenvector corresponding to $\lambda_{m}, A v_{m}=\lambda_{m} v_{m}$. It is a routine matter to verify that $u_{m}(t)$ has the correct asymptotic behavior near $t=0$ and that it satisfies the differential equation in the sense of Definition III-1.

Conversely, suppose $\left\{\lambda_{n}\right\}_{n=1}^{\infty}$ is admissible for uniqueness of strong solutions. Let, for $\delta \in(0, T)$,

$$
v_{n}(\delta) \equiv \int_{\delta}^{T} t^{1 / 2(1+k)} \psi_{n}(t) u(t) d t,
$$

where $u$ is the given strong solution to the FBVP. The vectors $v_{n}(\delta)$ are well defined in view of the strong continuity of $u$. Moreover, it is easy to check that

$$
t^{1 / 2(1+k)} \psi_{n}(t)= \begin{cases}O\left(t^{k}\right) & \text { as } t \rightarrow 0^{+}, k>0, \\ O(t) & \text { as } t \rightarrow 0^{+}, k<0\end{cases}
$$

From this one sees that the function $t^{1 / 2(1+k)} \psi_{n}(t)\|u(t)\|$ is integrable near $t=$ 0 . Consequently $\lim _{\delta \rightarrow 0}+v_{n}(\delta)$ exists in the strong topology on $B$. Let $v_{n}$ denote this limit. From integration theory in Banach space (Hille and Phillips [13, p. $62]$ ) and the assumption that $A$ is closed, we have that $v_{n}(\delta) \in D(A)$ and

$$
A v_{n}(\delta)=\int_{\delta}^{T} t^{1 / 2(1+k)} \psi_{n}(t) A u(t) d t
$$

Using (iv) of Definition III-1 we find that the strong limit as $\delta \rightarrow 0^{+}$of $A v_{n}(\delta)$ exists. Call this limit $y_{n}$. In view of the closedness of $A$ again, it follows that $v_{n} \in D(A)$ and $A v_{n}=y_{n}$.

Now fix $f \in B^{*}$. We have 


$$
\begin{aligned}
\left\langle f, A v_{n}(\delta)\right\rangle= & \int_{\delta}^{T} t^{1 / 2(1+k)} \psi_{n}(t)\langle f, A u(t)\rangle d t \\
& =-\int_{\delta}^{T} t^{1 / 2(1+k)} \psi_{n}(t)\left(\frac{d^{2}}{d t^{2}}\langle f, u(t)\rangle+\frac{k}{t} \frac{d}{d t}\langle f, u(t)\rangle\right) d t \\
& =t^{k}\left(t^{1 / 2(1-k)} \psi_{n}(t)\right)^{\prime}\langle f, u(t)\rangle-\left.t^{1 / 2(1+k)} \psi_{n}(t)\langle f, d u \mid d t\rangle\right|_{\delta} ^{T} \\
& +\lambda_{n} \int_{\delta}^{T} t^{1 / 2(1+k)} \psi_{n}(t)\langle f, u(t)\rangle d t .
\end{aligned}
$$

In view of the boundary conditions this leads to

$$
\left\langle f, A v_{n}\right\rangle=\lim _{\delta \rightarrow 0^{+}}\left\langle f, A v_{n}(\delta)\right\rangle=\lambda_{n} \lim _{\delta \rightarrow 0^{+}}\left\langle f, v_{n}(\delta)\right\rangle=\lambda_{n}\left\langle f, v_{n}\right\rangle .
$$

Therefore $\left\langle f, A v_{n}-\lambda_{n} v_{n}\right\rangle=0$ for all $n$ and all $f \in B^{*}$. From a corollary of the Hahn-Banach Theorem it follows that $A v_{n}-\lambda_{n} v_{n}=0$ for all $n$. Since $\lambda_{n} \notin$ $\sigma_{p}(A), v_{n}=0$ for all $n$. Thus, for any $f \in B^{*}$,

$$
0=\left\langle f, v_{n}\right\rangle=\int_{0}^{T} t^{1 / 2(1+k)} \psi_{n}(t)\langle f, u(t)\rangle d t
$$

It follows from the completeness properties of the Bessel functions that $\langle f, u(t)\rangle \equiv 0$ for $t \in(0, T]$ and $f \in B^{*}$. The same separation property of $B^{*}$ as above which follows from the Hahn-Banach Theorem permits us to conclude that $u(t) \equiv 0$.

IV. Other boundary conditions. Many of the arguments of the preceding section can be extended to other boundary conditions. The only modification being the choice of the sequence $\left\{\lambda_{n}\right\}_{n=1}^{\infty}$. We consider the general condition

$$
u^{\prime}(T)+\alpha u(T)=0 \text {. }
$$

The condition to be satisfied by the eigenfunctions $\psi_{n}(t)$ is

$$
\psi_{n}^{\prime}(T)+(\alpha+1 / 2(1-k) / T) \psi_{n}(T)=0 .
$$

Recall that $\psi_{n}(t)=J_{\sigma}(\sqrt{\lambda} t)$ where $\sigma=1 / 2(k-1)$ if $k>0$ and $\sigma=1 / 2(1-k)$ if $k<0$. This means the sequence of positive numbers $\lambda_{n}$ satisfy

$$
{\sqrt{\lambda_{n}}} T J_{\sigma}^{\prime}\left({\sqrt{\lambda_{n}}} T\right)+[\alpha T+1 / 2(1-k)] J_{\sigma}\left({\sqrt{\lambda_{n}}}_{n}\right)=0 .
$$

We shall prove the following theorems. In both cases $u$ is presumed to be a strong solution to (3.1) in the sense of Definition III-1 satisfying the regularity conditions near $t=0$ of Definition III-2. The sequence $\left\{\lambda_{n}\right\}_{n=1}^{\infty}$ will denote the positive (squares) of positive roots of (4.3). 
THEOREM IV-1. Let $k>0$ and $u:(0, T] \rightarrow D$ be as above and suppose $\dot{u}(T)+\alpha(T)=0$.

Then we have

(a) If $\alpha>0, u \equiv 0$ if and only if $\left\{\lambda_{n}\right\}_{n=1}^{\infty}$ is admissible for uniqueness in the sense of Definition II-1. (No $\lambda_{n} \in \sigma_{p}(A)$.)

(b) If $\alpha=0, u(t)=v$ a constant vector in $D(A)$ if and only if $\lambda_{n} \notin \sigma_{p}(A)$ for all $n$. The vector $v$ satisfies $A v=0$. Thus, in this case $u \equiv 0$ if and only if $0 \notin \sigma_{p}(A)$.

(c) If $\alpha<0, u(t)=t^{1 / 2(1-k)} I_{\sigma}(\beta t) v$ where $v \in D(A)$ satisfies $A v+\beta^{2} v=$ $0, I_{\sigma}(t)=i^{-\sigma} J_{\sigma}(i t)$ is the modified Bessel function and $i \beta$ is one of the conjugate imaginary roots of (4.3) if and only if $\lambda_{n} \notin \sigma_{p}(A)$ for all $n$. Moreover, $u(t) \equiv 0$ if and only if $-\beta^{2} \notin \sigma_{p}(A) .(\sigma=1 / 2(k-1)$ here. $)$

For the case $k<0$, we obtain a similar result. Here the natural breakoff point is $\alpha=(k-1) / T$. We have

TheOREM IV-2. Let $k<0$ and $u:(0, T] \rightarrow D$ be as above. Suppose $u$ satisfies $\dot{u}(T)+\alpha u(T)=0$. Then we have

(a) If $\alpha>(k-1) / T, u(t) \equiv 0$ if and only if the sequence $\left\{\lambda_{n}\right\}_{n=1}^{\infty}$ is admissible for uniqueness in the sense of Definition II-1. (Here $\sigma=1 / 2(1-k)$.)

(b) If $\alpha=(k-1) / T, u(t)=t^{1-k} v$ for some $v \in D(A)$ with $A v=0$ if and only if $\lambda_{n} \notin \sigma_{p}(A)$ for all $n$. In this case $u \equiv 0$ if and only if $0 \notin \sigma_{p}(A)$.

(c) If $\alpha<(k-1) / T, u(t)=t^{1 / 2(1-k)} I_{\sigma}(\beta t) v$ where $v \in D(A)$ solves $A v+$ $\beta^{2} v=0$ if and only if $\lambda_{n} \notin \sigma_{p}(A)$ for all $n$. (Here $I_{\sigma}, \beta$ are as in the preceding theorem.) Moreover $u(t) \equiv 0$ in this case if and only if $-\beta^{2} \notin \sigma_{p}(A)$.

The proofs of these results rest upon the following well-known property of Bessel functions [4], [14].

Consider the roots of

$$
\mu T J_{\sigma}^{\prime}(\mu T)+H J_{\sigma}(\mu T)=0,
$$

where $\sigma \geqslant-1 / 2$ and $H$ is a real constant and let $\sqrt{\lambda_{n}}=\mu_{n}$ denote the positive roots of (4.4).

(A) If $H>-\sigma / T$, then (4.4) has only the positive roots $\mu_{n}$, and the functions $\psi_{n}(t)=J_{\sigma}\left(\mu_{n} t\right)$ form a complete orthogonal set on $(0, T)$ with respect to the measure $t d t$.

(B) If $H=-\sigma / T,(4.4)$ has 0 as a root, and the family $\left\{t^{\sigma}, \psi_{1}(t), \ldots\right\}$ forms a complete orthogonal set on $(0, T)$ with respect to the measure $t d t$.

(C) If $H<-\sigma / T,(4.4)$ has a pair of imaginary roots $\pm i \beta$, and the family $\left\{I_{\sigma}(\beta t), \psi_{1}(t), \ldots\right\}$ forms a complete orthogonal family on $(0, T)$ with respect to the measure $t d t$. Here $I_{\sigma}(t)=i^{-\sigma} J_{\sigma}(i t)$ is the modified Bessel function of order $\sigma$. Note that it is strictly positive on $(0, T]$. 
If we set $H=\alpha+1 / 2(1-k) / T$, the conditions $H>-\sigma / T, H=-\sigma / T$ and $H<-\sigma / T$ become $\alpha>0, \alpha=0$ and $\alpha<0$, if $k>0, \sigma=1 / 2(k-1)$; and they become $\alpha>(k-1) / T, \alpha=(k-1) / T$ and $\alpha<(k-1) / T$ if $k<0$ and $\sigma=$ $1 / 2(1-k)$.

Now we can prove the two theorems. If $\alpha>0(k>0)$ or $\alpha>(k-1) / T$, $(k<0)$, the proof of (a) in either case is the same as that of Theorem III-1. The counterexamples, if $\lambda_{m} \in \sigma_{p}(A)$ are taken as in Theorem III-1.

Now consider the case $\alpha=0, k>0$. As in the other cases

$$
v_{n} \equiv \int_{0}^{T} t^{1 / 2(k-1)} \psi_{n}(t) u(t) \cdot t d t=0,
$$

for $u=1,2, \ldots$ provided that $\lambda_{n} \notin \sigma_{p}(A)$ for all $u=1,2, \ldots$. In this case, we must have, for every $f \in B^{*}$,

$$
\left\langle t^{1 / 2(k-1)} f, u(t)\right\rangle=c_{f} t^{1 / 2(k-1)}
$$

or $\langle f, u(t)\rangle=c_{f}$, a constant. Thus $u(t)=v$, a constant in $D(A)$. For if not, there are $t_{1}, t_{2} \in(0, T], t_{1} \neq t_{2}$ such that $u\left(t_{1}\right) \neq u\left(t_{2}\right)$. Thus there is an element $g \in B^{*}$ such that $c_{g}=\left\langle g, u\left(t_{1}\right)\right\rangle \neq\left\langle g, u\left(t_{2}\right)\right\rangle=c_{g}$. But this is impossible. Thus $u(t)=v$. Since $u$ takes values in $D(A), v \in D(A)$ as well. Introducing this solution into (3.2) we find that $\langle f, A v\rangle=0$ for all $f \in B^{*}$. Thus $A v=0$. The second statement of part (B) follows immediately. Then

If on the other hand some $\lambda_{m} \in \sigma_{p}(A)$, let $v_{m} \in D(A), A v_{m}=\lambda_{m} v_{m}$.

$$
u_{m}(t)=t^{1 / 2(1-k)} J_{1 / 2(k-1)}\left({\sqrt{\lambda_{m}}} t\right) v_{m}
$$

satisfies (3.2), $\left\langle f, \dot{u}_{m}(T)\right\rangle=0$ and the regularity conditions near $t=0$ of Definition III-2.

Now consider the case $\alpha=(k-1) / T, k<0$. In exactly the same manner as above $v_{n}=0$ if $\lambda_{n} \notin \sigma_{p}(A)$. Thus, for any $f \in D(A)$,

$$
t^{1 / 2(k-1)}\langle f, u(t)\rangle=c_{f} t^{1 / 2(1-k)}
$$

for some constant $c_{f}$. Thus $\left\langle f, t^{(k-1)} u(t)\right\rangle=c_{f}$. As in the preceding argument, this implies that $t^{k-1} u(t)=v$, a constant in $D(A)$ and hence $u(t)=t^{1-k} v$. This is a solution with $A v=0$ and it clearly satisfies $t\langle f, \dot{u}(T)\rangle+(k-1)\langle f, u(T)\rangle=$ 0 as well as the regularity requirements near $t=0$ demanded by Definition III-2 for $k<0$. The second statement of part (b) follows immediately. The necessity is again established by letting

$$
u_{m}(t)=t^{1 / 2(1-k)} J_{1 / 2(1-k)}\left(\sqrt{\lambda}_{m} t\right) v_{m}
$$

where $\lambda_{m} \in \sigma_{p}(A)$ and $A v_{m}=\lambda_{m} v_{m}, v_{m} \neq 0, v_{m} \in D(A)$. 
The proof of the third parts of these theorems are similar to those of parts (b). The only difference is that one must show that $t^{1 / 2(k-1)}\langle f, u(t)\rangle=$ $c_{f} I_{\sigma}(\beta t)$ implies that $u(t)=t^{1 / 2(1-k)} I_{\sigma}(\beta t) v$ for $v \in D(A)$ with $A v+\beta^{2} v=0$. To see this we observe that $I_{\sigma}>0$ on $(0, T]$ so that

$$
\left\langle f,\left[t^{1 / 2(k-1)} / I_{\sigma}(\beta t)\right] u(t)\right\rangle=c_{f},
$$

for all $f \in B^{*}$. Then, it follows as before, from the Hahn-Banach Theorem, that the second argument in $\langle$,$\rangle above is a constant which must be in D(A)$ since $u(t)$ is for $t \in(0, T]$. The fact that $u(t)$ satisfies (3.2) leads to $\left\langle f, A v+\beta^{2} v\right\rangle=$ 0 for all $f \in B^{*}$ so again $A v+\beta^{2} v=0$.

The necessity of the conditions $\lambda_{m} \notin \sigma_{p}(A)$ follows as in parts (a), (b). We omit details.

V. Weak solutions. In this section we examine the question of uniqueness for weak solutions of (1.1) and (1.3). In order to do this we shall need some additional technical requirements on the operator $A$. In fact, instead of (1.1) we shall consider the more general

$$
P u_{t t}+A u=0
$$

where $P$ and $A$ are unbounded operators on $D \subset B$, a dense linear subspace and formulate and prove our result for weak solutions to (1.3) and content ourselves with a formulation and statement of the analogous results for (5.1).

DEFINITION V-1. We say a sequence $\left\{\lambda_{n}\right\}_{n=1}^{\infty}$ is admissible for uniqueness of weak solutions if $A^{*}-\lambda_{n}$ has dense range in $B^{*}$. (In complex Banach space we would replace this condition by $A^{*}-\bar{\lambda}_{n}$ has dense range.)

Clearly Definition II-1 is weaker than Definition V-1. That they are not equivalent in general can be seen from the following example due to Robert Sine. We let $B=C[0,1]$, the continuous functions on $[0,1]$ and $\|f\|=$ $\sup _{t \in[0,1]}|f(t)|$. Define $A: B \rightarrow B$ by $A f(t)=t f(t)$. $A$ is a bounded operator with no eigenvalues. The dual space $B^{*}$ of $B$ is the set of regular Borel measures $m$. The adjoint, $A^{*}$ acts on measures by the following

$$
\langle m, A f\rangle=\int_{[0,1]} t f(t) d m(t)=\int_{[0,1]} f(t) t d m(t)=\langle t m, f\rangle,
$$

so that $A^{*} m=t m$. Now $0 \notin \sigma_{p}(A)$ but the range of $A^{*}$ is not dense in $B^{*}$. (The norm on $B^{*}$ is the total variation of $m\left(\in B^{*}\right)$.) To see this, we observe that $\operatorname{tm}\{0\}=0$. Let $\delta$ be point mass at 0 . Then clearly $\|\delta-t m\|^{*} \geqslant 1$ where $\|m\| *$ is the total variation of $m$.

However, if $B$ is a Hilbert space and $A^{* *}=A$, then one can easily show that $\lambda \notin \sigma_{p}(A)$ if and only if $A-\lambda$ has dense range. 
Definition V-2. We say $u:(0, T] \rightarrow D(A) \subset B$ is a weak solution to the first boundary value problem for (3.1) if

(i) $u$ is strongly continuous on $(0, T]$.

(ii) $u(T)=0$.

(iii) (a) if $k>0, t^{k}\|u(t)\| \in L^{1}(0, T)$;

(b) if $k<0, t\|u(t)\| \in L^{1}(0, T)$.

(iv)

$$
0=\int_{0}^{T}\left\langle v^{\prime \prime}(t)-(k v(t) / t)^{\prime}+A^{*} v(t), u(t)\right\rangle d t
$$

for all $v:(0, T] \rightarrow D\left(A^{*}\right)$ satisfying

$(\alpha) v$ is twice strongly continuously differentiable.

(B) $v(T)=0$.

(r) If $k>0$, then $w(t)=t^{-k} v(t)$ satisfies

$$
\sup _{(0, T]}\left\{\|w(t)\|,\left\|w^{\prime \prime}(t)-k t^{-1} w^{\prime}(t)\right\|\right\}<\infty .
$$

If $k<0, w(t)=t^{-1} v(t)$ satisfies $(\gamma)$ above.

$(\delta)$ In either case $\left\|A^{*} w(t)\right\|$ is bounded on $(0, T]$.

It is now an easy matter to prove

THEOREM V-1. Let $u:(0, T] \rightarrow B$ be a weak solution of the first boundary value problem above. Let $\left\{\lambda_{n}\right\}_{n=1}^{\infty}$ be the squares of the positive roots of $J_{\sigma}(\mu T)=0$ for $\sigma=1 / 2(k-1)$ if $k>0$ and $\sigma=1 / 2(1-k)$ if $k<0$. If $\left\{\lambda_{n}\right\}_{n=1}^{\infty}$ is admissible for uniqueness in the sense of the above definition, then $u \equiv 0$.

Proof. Fix $f \in D\left(A^{*}\right)$ and let

$$
\sigma_{n}(t)=t^{1 / 2(1+k)} J_{\sigma}\left(\sqrt{\lambda}_{n} t\right) \cdot f=t^{1 / 2(1+k)} \psi_{n}(t) f .
$$

It is easy to verify that $\sigma_{n}$ is admissible in (5.2). Inserting $\sigma_{n}$ in (5.2) we find that

$$
0=\int_{0}^{T} t^{1 / 2(1+k)} \psi_{n}(t)\left\langle-\lambda_{n} f+A^{*} f, u(t)\right\rangle d t .
$$

Again setting

$$
v_{n}=\int_{0}^{T} t^{1 / 2(1+k)} \psi_{n}(t) u(t) d t
$$

(the existence of $v_{n}$ follows exactly as in the case of strong solutions) we may rewrite (5.3.1) as

$$
0=\left\langle A^{*} f-\lambda_{n} f, v_{n}\right\rangle
$$

because 


$$
\begin{aligned}
\left\langle A^{*} f, v_{n}\right\rangle & =\int_{0}^{T} t^{1 / 2(1+k)} \psi_{n}(t)\left\langle A^{*} f, u(t)\right\rangle d t \\
& =\lambda_{n} \int_{0}^{T} t^{1 / 2(1+k)} \psi_{n}(t)\langle f, u(t)\rangle d t \\
& =\lambda_{n}\left\langle f, v_{n}\right\rangle .
\end{aligned}
$$

Since $A^{*}-\lambda_{n}$ has dense range, we obtain, for each fixed $n, v_{n}=0$. The remainder of the argument is the same as that used in Theorem III-1.

REMARK. Although we obtain uniqueness of solutions in a much wider class, we no longer assert the necessity of the condition that $A^{*}-\lambda_{n}$ have dense range for all $n$. Moreover, we had to assert more about the structure of $A$.

We now take up the question of weak solutions to

$$
\begin{gathered}
P \frac{d^{2} u}{d t^{2}}+A u(t)=0, \quad t \in(0, T), \\
u(0)=u(T)=0 .
\end{gathered}
$$

We assume that the operators $P, A$ are defined on a dense domain $D$ and that the adjoint operator $P^{*}, A^{*}$ are defined on a dense subdomain $D^{*} \subset B^{*}$.

Definition V-3. We say $u:[0, T] \rightarrow D$ is a weak solution to (5.4), (5.5) if $u$ is strongly continuous on $[0, T]$ and

$$
\int_{0}^{T}\left\langle P^{*} \psi^{\prime \prime}(t)+A^{*} \psi(t), u(t)\right\rangle d t=0
$$

for every $\psi:[0, T] \rightarrow D^{*}$ such that

(i) $\psi$ is twice strongly continuously differentiable in the norm of $B^{*}$,

(ii) $\psi^{\prime \prime}$ takes values in $D^{*}$,

(iii) $\psi(0)=\psi(T)=0$.

We say a sequence $\left\{\lambda_{n}\right\}_{n=1}^{\infty}$ is admissible for uniqueness of weak solutions of (5.4), (5.5), if for each $n, P^{*}-\lambda_{n} A^{*}$ has dense range in $B^{*}$.

THEOREM V-2. If $\lambda_{n} \equiv n^{2} \pi^{2} / T^{2}, n=1,2, \ldots$, forms a sequence which is admissible for uniqueness of weak solutions of (5.4), (5.5), then $u \equiv 0$ is the only weak solution of (5.4), (5.5) in the sense of Definition V-3.

The proof of this result is very similar to that of the preceding result. We simply take $\psi_{n}(t)=\sin (n \pi t / T) f$ where $f \in D^{*}$ and insert this in (5.6). We find that

$$
0=\int_{0}^{T}\left\langle P^{*} f-\lambda_{n} A^{*} f, u(t)\right\rangle \sin {\sqrt{\lambda_{n}} t d t}
$$

since $P^{*} f$ and $A^{*} f$ are bounded linear functionals, we may set

$$
v_{n}=\int_{0}^{T} \sin (n \pi t / T) u(t) d t
$$


and argue from (5.7) that

$$
\left\langle P^{*} f-\lambda_{n} A^{*} f, v_{n}\right\rangle=0
$$

Thus $v_{n}=0$ and another application of the Hahn-Banach Theorem as well as the completeness properties of $\{\sin (n \pi t / T)\}_{n=1}^{\infty}$ gives the desired result.

REMARK. In the case in which $B$ is a Hilbert space and $P^{* *}=P, A^{* *}=A$, we can show that the conditions on $\left\{\lambda_{n}\right\}_{n=1}^{\infty}$, are necessary as well as sufficient in Theorems V-1 and V-2 for weak solutions. For in this case, if $A^{*}-\lambda_{n}$ (or $\left.P^{*}-\lambda_{n} A^{*}\right)$ does not have dense range, then there is $x \in B$ such that $x \in D(A)$ (or $D$ ), and such that $x \neq 0$ satisfying $A x-\lambda_{n} x=0$ (or $P x-\lambda_{n} A x=0$ ). The strong solutions given by $u_{n}(t)=t^{1 / 2(1-k)} J_{\sigma}\left(\sqrt{\lambda}_{n} t\right) x\left(u_{n}(t)=\sin (n \pi t / T) x\right)$ are then nontrivial weak solutions as well and the necessity is thus established.

VI. It is clear from the preceding discussion that our results apply equally well to the Euler-Poisson-Darboux equation $(A=-\Delta)$ or the GASPT (generalized axially symmetric potential theory) equation $(A=\Delta)$. Other generalizations are possible. Consider for example an equation of the form

$$
\sum_{i=1}^{n} \frac{\partial^{2} u}{\partial t_{i}^{2}}+\frac{k_{i}}{t_{i}} \frac{\partial}{\partial t_{i}}+A u=0, \quad \vec{t} \in\left(t_{1}, \ldots, t_{n}\right) \in{\underset{X}{X=1}}_{x}^{n}\left(0, T_{i}\right]
$$

where $u: \times_{i=1}^{n}\left(0, T_{i}\right] \rightarrow D(A) \subset B$. The numbers $T_{i}$ are all finite and the constants $k_{i}$ are fixed, given quantities. Equation (6.1) may be referred to as the generalized $n$-axially symmetric potential theory equation.

Uniqueness theorems for (6.1) may be obtained along the following lines. As a boundary condition appropriate to (6.1) we might take $u\left(\hat{t}_{i}, T_{i}\right)=0, i=$ $1, \ldots, n$, where $\left(\hat{t}_{i}, T_{i}\right)=\left(t_{1}, \ldots, t_{i-1}, T_{i}, t_{i+1}, \ldots, t_{n}\right)$. We would set

$$
v_{\vec{m}}=\int_{0}^{T_{1}} \cdots \int_{0}^{T_{n}} \prod_{i=1}^{n}\left[t_{i}^{1 / 2\left(1+k_{i}\right)} J_{\sigma_{i}}\left(v^{\lambda_{m_{i}}} t_{i}\right)\right] u(\vec{t}) d t_{n} \cdots d t_{1}
$$

where $\vec{m}=\left(m_{1}, \ldots m_{n}\right)$ and $m_{i}=1,2,3, \ldots, i=1,2,3, \ldots, n$. The condition, necessary and sufficient for uniqueness, would then be $\sum_{i=1}^{n} \lambda_{m} \notin \sigma_{p}(A)$ for any $m$-tuple $\left(m_{1}, \ldots, m_{n}\right), m_{i}$ a positive integer.

Here $\sigma_{i}=1 / 2\left(k_{i}-1\right)$ if $k_{i}>0$ and $\sigma_{i}=1 / 2\left(1-k_{i}\right)$ if $k_{i}<0$. The regularity conditions which must be prescribed as each $t_{i} \rightarrow 0^{+}$when the others are held fixed are the same as those given in Theorem III-1 and will depend on the sign of $k_{i}$.

Our results concerning this problem contain those of Young [12] as a special case. The special case $k_{i}=0, i=1, \ldots, n$ is a generalization of the ultrahyperbolic equation treated in [5]. 
ACKNOWLedgments. The authors thank Professors Robert Sine and Richard Weinacht for their helpful comments.

The following list of references is not intended to be exhaustive.

\title{
REFERENCES
}

1. A. I. Abdul-Latif and J. B. Diaz, Dirichlet, Neumann and mixed boundary value problems for the wave equation $u_{x x}-u_{y y}=0$ for a rectangle, Applicable Anal. 1 (1971), $1-12$. MR 43 \#6603.

2. D. G. Bourgin, The Dirichlet problem for the damped wave equation, Duke Math. J. 7 (1940), 97-120. MR 2, 290.

3. D. G. Bourgin and R. Duffin, The Dirichlet problem for the vibrating string equation, Bull. Amer. Math. Soc. 45 (1939), 851-858. MR 1, 120.

4. F. Bowman, Introduction to Bessel functions, Dover, New York, 1958. MR 20 \#4007.

5. J. B. Diaz and E. C. Young, Uniqueness of solutions of certain boundary value prob. lems for ultrahyperbolic equations, Proc. Amer. Math. Soc. 29 (1971), 569-574. MR 44 \#626.

6. D. R. Dunninger and E. C. Zachmanoglou, The condition for uniqueness of the Dirichlet problem for hyperbolic equations in cylindrical domains, J. Math. Mech. 18 (1969), 763766.

7. D. R. Dunninger and H. A. Levine, Uniqueness criteria for solutions of abstract boundary value problems (manuscript).

8. D. R. Dunninger and R. J. Weinacht, Improperly posed problems for singular equations of the fourth order, Applicable Anal. 4 (1975), 331-341.

9. D. W. Fox and C. Pucci, The Dirichlet problem for the wave equation, Ann. Math. Pura Appl. (4) 46 (1958), 155-182. MR 21 \#3653.

10. F. John, The Dirichlet problem for a hyperbolic equation, Amer. J. Math. 63 (1941), 141-154. MR 2, 204.

11. E. C. Young, Uniqueness theorems for certain improperly posed problems, Bull. Amer. Math. Soc. 77 (1971), 253-256. MR 42 \#3426.

12. Uniqueness of solutions for the Dirichlet problem for singular ultrahyper. bolic equations, Proc. Amer. Math. Soc. 36 (1972), 130-136.

13. E. Hille and R. S. Phillips, Functional analysis and semi-groups, rev. ed., Amer. Math. Soc. Colloq. Publ., vol. 31, Amer. Math. Soc., Providence, R. I., 1957. MR 19, 664.

14. G. N. Watson, Theory of Bessel functions, Cambridge Univ. Press, Cambridge; Macmillan, New York, 1922.

\author{
DEPARTMENT OF MATHEMATICS, MICHIGAN STATE UNIVERSITY, EAST LAN- \\ SING, MICHIGAN 48823
}

DEPARTMENT OF MATHEMATICS, UNIVERSITY OF RHODE ISLAND, KINGSTON, RHODE ISLAND 02881 